\title{
ANTIMICROBIAL SUSCEPTIBILITY OF RIEMERELLA ANATIPESTIFER STRAINS ISOLATED FROM GEESE AND DUCKS IN HUNGARY
}

\author{
Éva GYURIS ${ }^{1}$, Enikő WEHMANN ${ }^{2}$, Katalin CZEIBERT ${ }^{1}$ and Tibor MAGYAR ${ }^{2 *}$ \\ ${ }^{1}$ Laboratory of Domestic Mammal, Wildlife and Poultry Diseases, National Food Chain \\ Safety Office - Veterinary Diagnostic Directorate, Budapest, Hungary; ${ }^{2}$ Institute for \\ Veterinary Medical Research, Centre for Agricultural Research, Hungarian Academy of \\ Sciences, P.O. Box 18, H-1581 Budapest, Hungary
}

(Received 14 November 2016; accepted 4 April 2017)

\begin{abstract}
Riemerella anatipestifer causes anatipestifer disease in many avian species. A total of 185 R. anatipestifer strains isolated in Hungary between 2000 and 2014 from geese and ducks were tested against 13 antibiotics (ampicillin, doxycycline, enrofloxacin, erythromycin, florfenicol, flumequine, gentamicin, penicillin, spectinomycin, streptomycin, sulphamethoxazole-trimethoprim, sulphonamide compounds, and tetracycline) by the Kirby-Bauer disk diffusion method. The majority of the strains were susceptible to florfenicol (97.9\%), ampicillin (95.1\%), penicillin (93\%), sulphamethoxazole-trimethoprim (92.4\%), and spectinomycin (86.5\%). The highest resistance rates were observed for flumequine, tetracycline, erythromycin and streptomycin $(94 \%, 91.4 \%, 75.1 \%$ and $71.4 \%$ resistance, respectively). The resistance patterns showed some variation depending on the geographical origin of the strains. The average rate of extensive drug resistance was $30.3 \%$, and its proportion tended to increase in the period examined.
\end{abstract}

Key words: Riemerella anatipestifer, antimicrobial susceptibility

Anatipestifer disease caused by Riemerella anatipestifer infection is prevalent in all countries where intensive goose and duck production is practised. It causes significant economic losses through high mortality, reduced growth rate and the costs of treatment and prevention (Ruiz and Sandhu, 2013). Since its first description in geese by Riemer (1904), the disease has been reported regularly, but the taxonomic position of the aetiological agent remained uncertain for many years (Hendrickson and Hilbert, 1932; Bruner and Fabricant, 1954; Breed et al., 1957). Finally Segers et al. (1993) named the agent $R$. anatipestifer and suggested its transfer to a separate genus (Riemerella) based on phenotypic and genotypic characteristics.

Riemerella anatipestifer causes anatipestifer disease primarily in young, 2to 8-week-old goslings and ducklings; however, severe losses have been reported

*Corresponding author; E-mail: magyar.tibor@agrar.mta.hu; Phone: 0036 (1) 467-4092 
in turkey flocks as well (Helfer and Helmboldt, 1977; Smith et al., 1987; Frommer et al., 1990). The disease has also been described in chickens, pheasants, guinea fowl, quails, gulls, budgerigars, and wild waterfowl (Bruner et al., 1970; Karstad et al., 1970; Rosenfeld, 1973; Pascucci et al., 1989; Hinz et al., 1998).

The clinical signs of anatipestifer disease include nasal discharge, coughing, sinusitis, diarrhoea and neural signs such as torticollis, head tremor and lameness (Bisgaard et al., 2008; Fulton and Rimler, 2010). The gross lesions are similar in different avian species and include a slightly enlarged spleen, fibrinous pericarditis, perihepatitis, airsacculitis, catarrhal rhinitis, pneumonia, purulent arthritis, catarrhal enteritis, as well as caseous exudate in the oviducts, skin necrosis and serous-fibrinous meningitis (Dougherty et al., 1955; Smith et al., 1987; Bisgaard et al., 2008). The disease may persist for two weeks without appropriate treatment, and mortality may vary from $10 \%$ to $75 \%$ (Ruiz and Sandhu, 2013).

In Hungary, anatipestifer disease was first described by Bitay et al. (1979) in ducks, while Ivanics et al. (1996) reported its first occurrence in geese.

Twenty-one serotypes of $R$. anatipestifer are known to date, and no crossprotection has been observed between the different serotypes. In addition, more than one serotype can be present in the same farm (Ruiz and Sandhu, 2013). These facts underline the importance of antibiotics in controlling anatipestifer disease.

The aim of this study was to determine the antibiotic susceptibility patterns of $185 R$. anatipestifer strains against 13 antibiotics.

\section{Materials and methods}

\section{Bacterial strains}

A total of $185 R$. anatipestifer strains were isolated from 2000 to 2014 from 100 flocks of geese or ducks located at 48 settlements of six counties in Hungary (Fig. 1).

All strains were recovered from 5- to 140-day-old birds that had died of anatipestifer disease. Samples taken from the liver, brain, pericardium or lung were cultured on Columbia agar plates (Biolab, Budapest, Hungary) supplemented with $5 \%$ defibrinated sheep blood at $37{ }^{\circ} \mathrm{C}$ for $24 \mathrm{~h}$ in an atmosphere containing $5 \% \mathrm{CO}_{2}$. A total of 158 strains originated from geese and 27 strains from ducks. The strains were identified by colony morphology, Gram staining and a species-specific polymerase chain reaction (PCR) (Kardos et al., 2007). All isolates were stored at $-70^{\circ} \mathrm{C}$ until analysed. Table 1 shows the properties of the $R$. anatipestifer strains used in this study. 


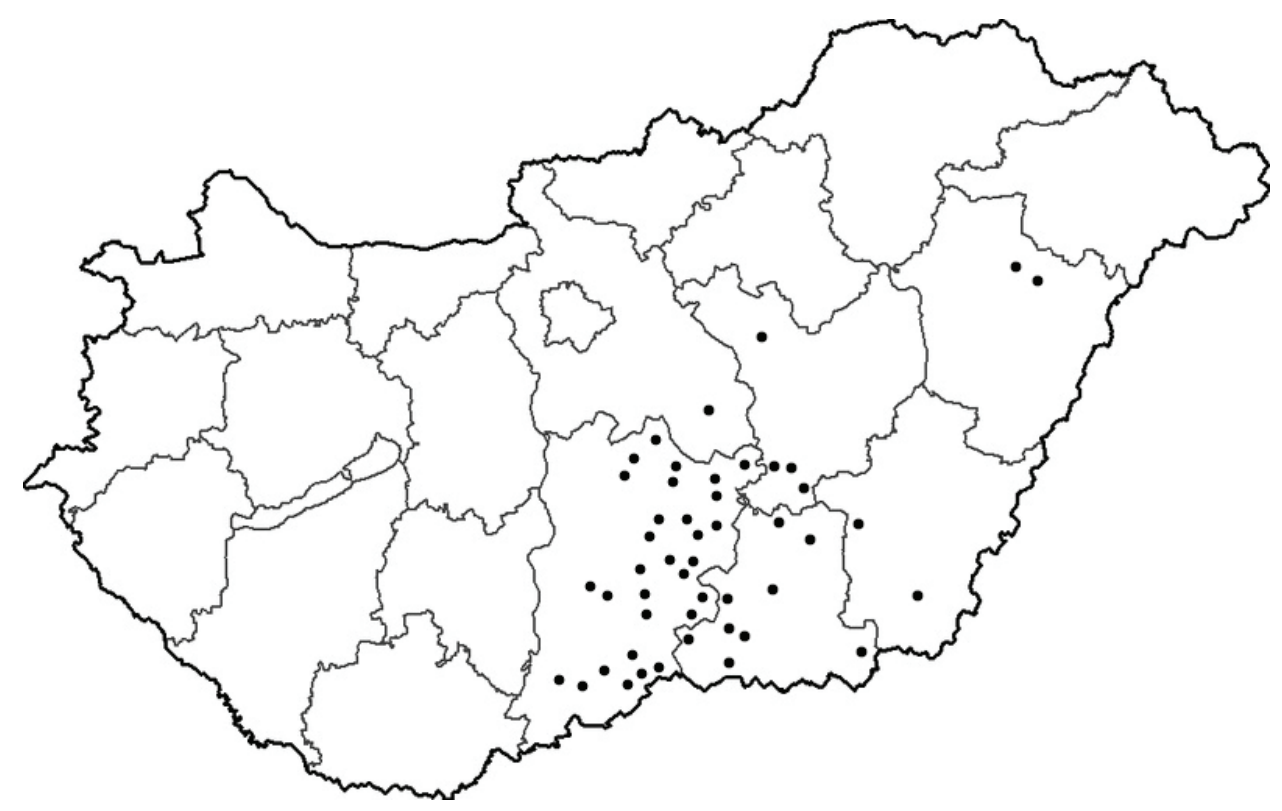

Fig. 1. Geographic origin of the Riemerella anatipestifer strains used in this study

\section{Susceptibility testing}

Antibiotic susceptibility was assessed by the Kirby-Bauer disk diffusion method. The isolates were tested for susceptibility to 13 antibiotics: ampicillin $(10 \mu \mathrm{g})$ [equivalent to the recently used amoxicillin $(10 \mu \mathrm{g})]$, doxycycline $(30 \mu \mathrm{g})$, enrofloxacin $(5 \mu \mathrm{g})$, erythromycin $(15 \mu \mathrm{g})$, florfenicol $(30 \mu \mathrm{g})$, flumequine $(30 \mu \mathrm{g})$, gentamicin $(10 \mu \mathrm{g})$, penicillin (10 IU), spectinomycin $(100 \mu \mathrm{g})$, streptomycin $(10 \mu \mathrm{g})$, sulphamethoxazole-trimethoprim $(23.75+1.25 \mu \mathrm{g})$, sulphonamide compounds $(300 \mu \mathrm{g})$, and tetracycline $(30 \mu \mathrm{g})$ (Biolab, Budapest, Hungary).

The tests were carried out according to the guidelines of the Clinical and Laboratory Standards Institute (CLSI, 2013, 2015). Strains were grown on Columbia agar plates supplemented with $5 \%$ defibrinated sheep blood at $37{ }^{\circ} \mathrm{C}$ in $5 \% \mathrm{CO}_{2}$ atmosphere. Colonies were suspended in $0.85 \%$ saline, and the turbidity was adjusted to $0.5 \mathrm{McFarland}$ standard. The disk diffusion analysis was performed on Mueller-Hinton agar (Biolab, Budapest, Hungary) enriched with 5\% sheep blood. Inhibitory zone diameters were measured after $24 \mathrm{~h}$ of incubation. The resistance breakpoints (Table 2) were interpreted according to the criteria provided by CLSI documents M100-S21 and VET01S (CLSI, 2013, 2015) and the National Food Chain Safety Office, Veterinary Diagnostic Directorate (Budapest, Hungary). 
Table 1

Properties of the Riemerella anatipestifer strains used in this study

\begin{tabular}{|c|c|c|c|}
\hline Geographic origin (county) & Host & Year of isolation & Number \\
\hline \multirow{13}{*}{ Bács-Kiskun } & goose & 2000 & 8 \\
\hline & & 2006 & 11 \\
\hline & & 2010 & 24 \\
\hline & & 2011 & 12 \\
\hline & & 2012 & 28 \\
\hline & & 2013 & 21 \\
\hline & & 2014 & 24 \\
\hline & duck & 2006 & 3 \\
\hline & & 2010 & 1 \\
\hline & & 2011 & 7 \\
\hline & & 2012 & 2 \\
\hline & & 2013 & 6 \\
\hline & & 2014 & 3 \\
\hline \multirow{2}{*}{ Békés } & goose & 2014 & 2 \\
\hline & duck & - & - \\
\hline \multirow{7}{*}{ Csongrád } & goose & 2010 & 3 \\
\hline & & 2011 & 4 \\
\hline & & 2012 & 2 \\
\hline & & 2013 & 1 \\
\hline & & 2014 & 2 \\
\hline & duck & 2013 & 1 \\
\hline & & 2014 & 2 \\
\hline \multirow{4}{*}{ Hajdú-Bihar } & goose & 2009 & 1 \\
\hline & & 2010 & 1 \\
\hline & & 2011 & 1 \\
\hline & duck & 2014 & 1 \\
\hline \multirow{4}{*}{ Jász-Nagykun-Szolnok } & goose & 2004 & 1 \\
\hline & & 2010 & 1 \\
\hline & & 2013 & 5 \\
\hline & duck & 2013 & 1 \\
\hline \multirow{4}{*}{ Pest } & goose & 2012 & 1 \\
\hline & & 2013 & 1 \\
\hline & & 2014 & 4 \\
\hline & duck & - & - \\
\hline
\end{tabular}


Table 2

Resistance breakpoints of the antibiotics used in this study

\begin{tabular}{lccc}
\hline & \multicolumn{3}{c}{ Zone diameter (mm) } \\
\cline { 2 - 4 } Antibiotic & \multicolumn{3}{c}{ Interpretive criteria } \\
\cline { 2 - 4 } & Susceptible & Intermediate & Resistant \\
\hline Penicillin & $\geq 24$ & - & $\leq 23$ \\
Ampicillin & $\geq 24$ & - & $\leq 23$ \\
Gentamicin & $\geq 15$ & $13-14$ & $\leq 12$ \\
Streptomycin & $\geq 15$ & $12-14$ & $\leq 11$ \\
Spectinomycin & $\geq 17$ & 16 & $\leq 15$ \\
Enrofloxacin & $\geq 23$ & $17-22$ & $\leq 16$ \\
Flumequine & $\geq 24$ & $20-23$ & $\leq 19$ \\
Tetracycline & $\geq 23$ & - & $\leq 22$ \\
Doxycycline & $\geq 23$ & - & $\leq 22$ \\
Erythromycin & $\geq 23$ & $14-22$ & $\leq 13$ \\
Sulphonamide compounds & $\geq 17$ & $13-16$ & $\leq 12$ \\
Sulphamethoxazole-trimethoprim & $\geq 16$ & $11-15$ & $\leq 10$ \\
Florfenicol & $\geq 19$ & $15-18$ & $\leq 14$ \\
\hline
\end{tabular}

\section{Results}

The majority of the strains were susceptible to florfenicol (97.9\%), ampicillin (95.1\%), penicillin (93\%), sulphamethoxazole-trimethoprim $(92.4 \%)$ and spectinomycin (86.5\%). Enrofloxacin and sulphonamide compounds proved to be less effective ( $26 \%$ and $27 \%$ resistance, respectively), although the rate of intermediate susceptibility was unusually high for enrofloxacin (60\%). Approximately $50 \%$ of the strains were resistant to gentamicin and doxycycline. The highest resistance rates were observed for flumequine, tetracycline, erythromycin and streptomycin (94\%, 91.4\%, 75.1\% and 71.4\% resistance, respectively) (Fig. 2).

Changes in the antibiotic resistance profiles of the strains over time were also evaluated (Table 3). A tendency of increase was noted in the rate of resistance to erythromycin, florfenicol, spectinomycin and streptomycin from 2000, sulphonamide compounds from 2004, and doxycycline from 2009. The rate of resistance to other antibiotics showed a variable tendency.

We examined the resistance pattern of four strains isolated within three weeks from one outbreak in a farm (Table 4). The strains displayed an expanding resistance pattern, showing resistance to $4,4,5$ and finally 7 antibiotics out of the 13 antibiotics examined.

Strains isolated from the north-western part of Bács-Kiskun County showed the highest resistance rate to doxycycline, gentamicin, spectinomycin, sulphamethoxazole-trimethoprim, sulphonamide compounds and tetracycline. 
Strains collected from the south-western part of Bács-Kiskun County possessed the lowest resistance rate to flumequine, gentamicin, spectinomycin and streptomycin (Table 5).

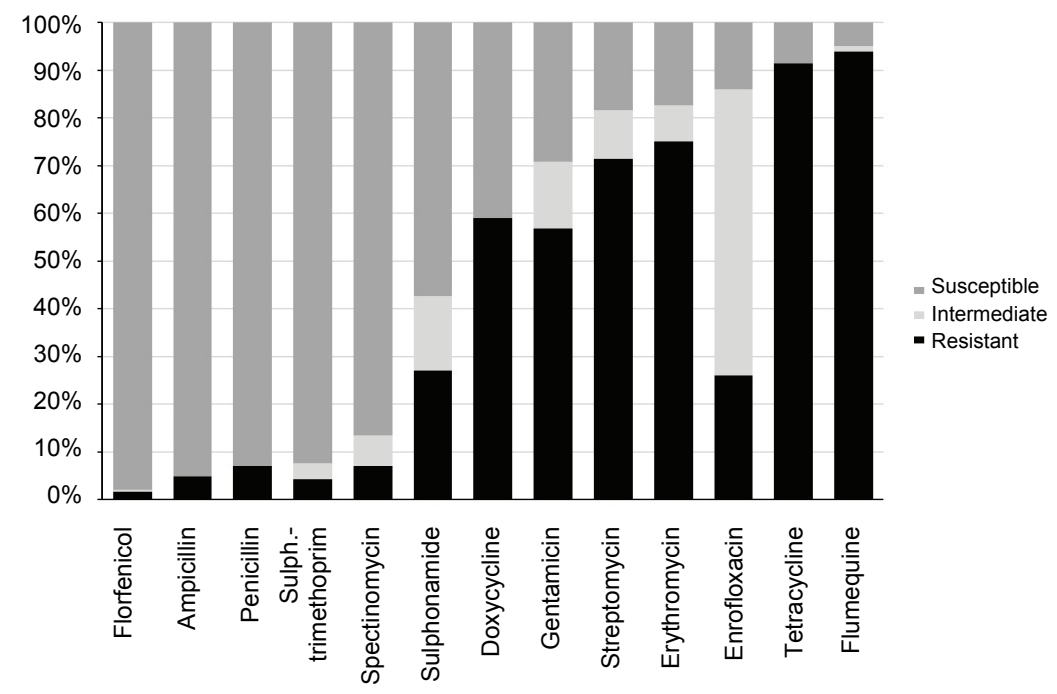

Fig. 2. Antimicrobial susceptibility of 185 Riemerella anatipestifer strains tested against 13 different antibiotics

Strains non-susceptible to at least one agent in all but two or fewer antimicrobial categories were defined as extensively drug-resistant (XDR) strains. Non-susceptibility refers to either a resistant, intermediate or non-susceptible result obtained from in vitro antimicrobial susceptibility testing (Magiorakos et al., 2012). The average rate of extensive drug resistance was $30.3 \%$ for all isolates. No XDR strain was found in 2000. Isolates collected between 2004 and 2006 showed $6.6 \%$ extensive drug resistance. The proportion of extensive drug resistance was $38.7 \%$ for strains isolated from 2009 to 2010 . Isolates collected from 2011, 2012, 2013 and 2014 showed an increasing tendency of extensive drug resistance: $20.8 \%, 18 \%, 30.5 \%$ and $55.3 \%$, respectively.

The rate of extensive drug resistance showed some variance according to the geographical origin of the strains. The proportion of extensive drug resistance was above average for strains isolated from the north-western part of BácsKiskun County and Csongrád County: $36.3 \%$ and 40\%, respectively. Isolates collected from the north-eastern and south-western part of Bács-Kiskun County showed $31.4 \%$ and $31.2 \%$ extensive drug resistance, respectively. The proportion of extensive drug resistance among strains isolated from the south-eastern part of Bács-Kiskun County was below average (28.3\%).

The proportion of XDR strains isolated from geese and ducks was $31 \%$ and $26 \%$, respectively. The rate of resistance to different antibiotics was irrespective of the host species. 


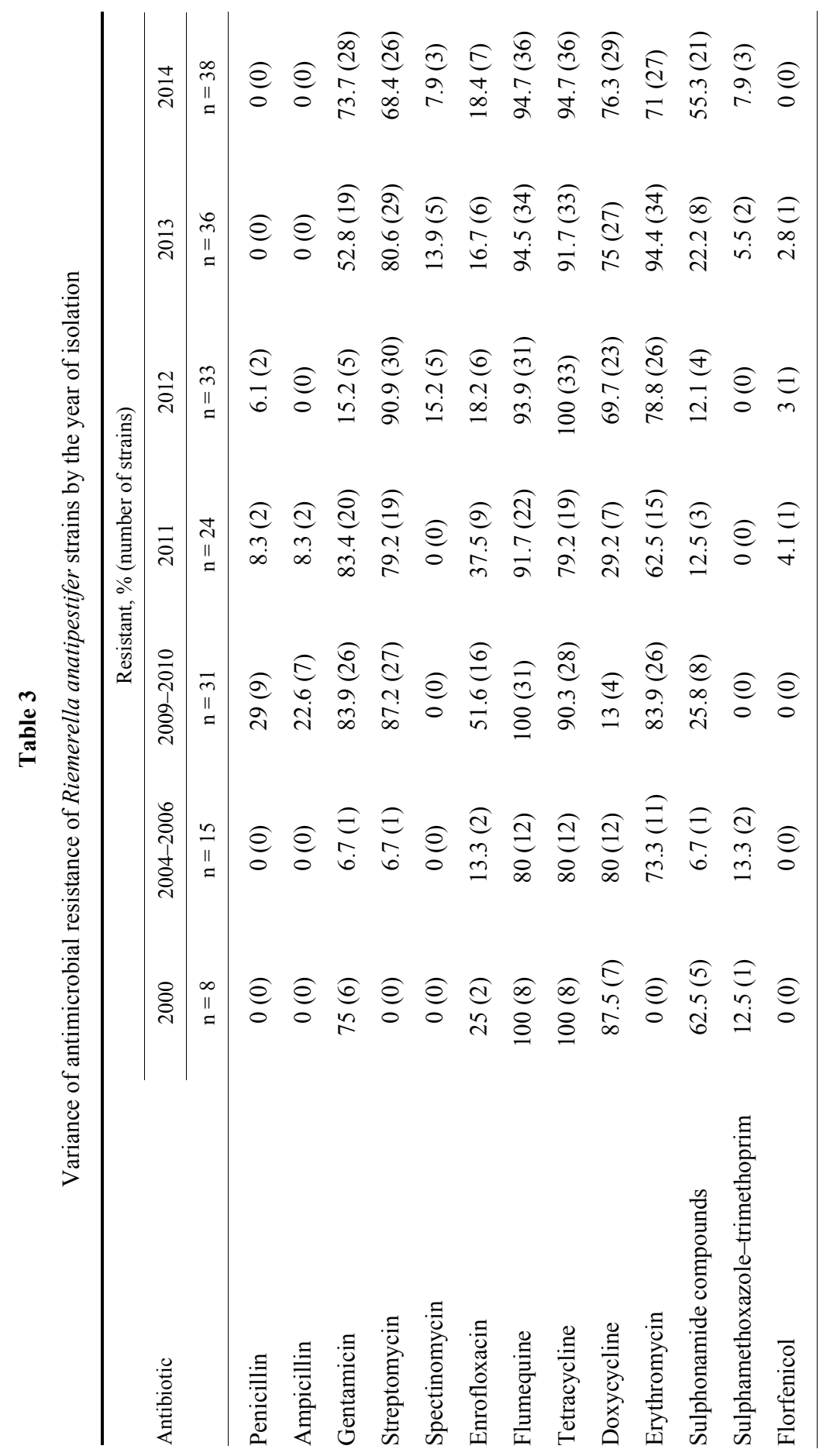




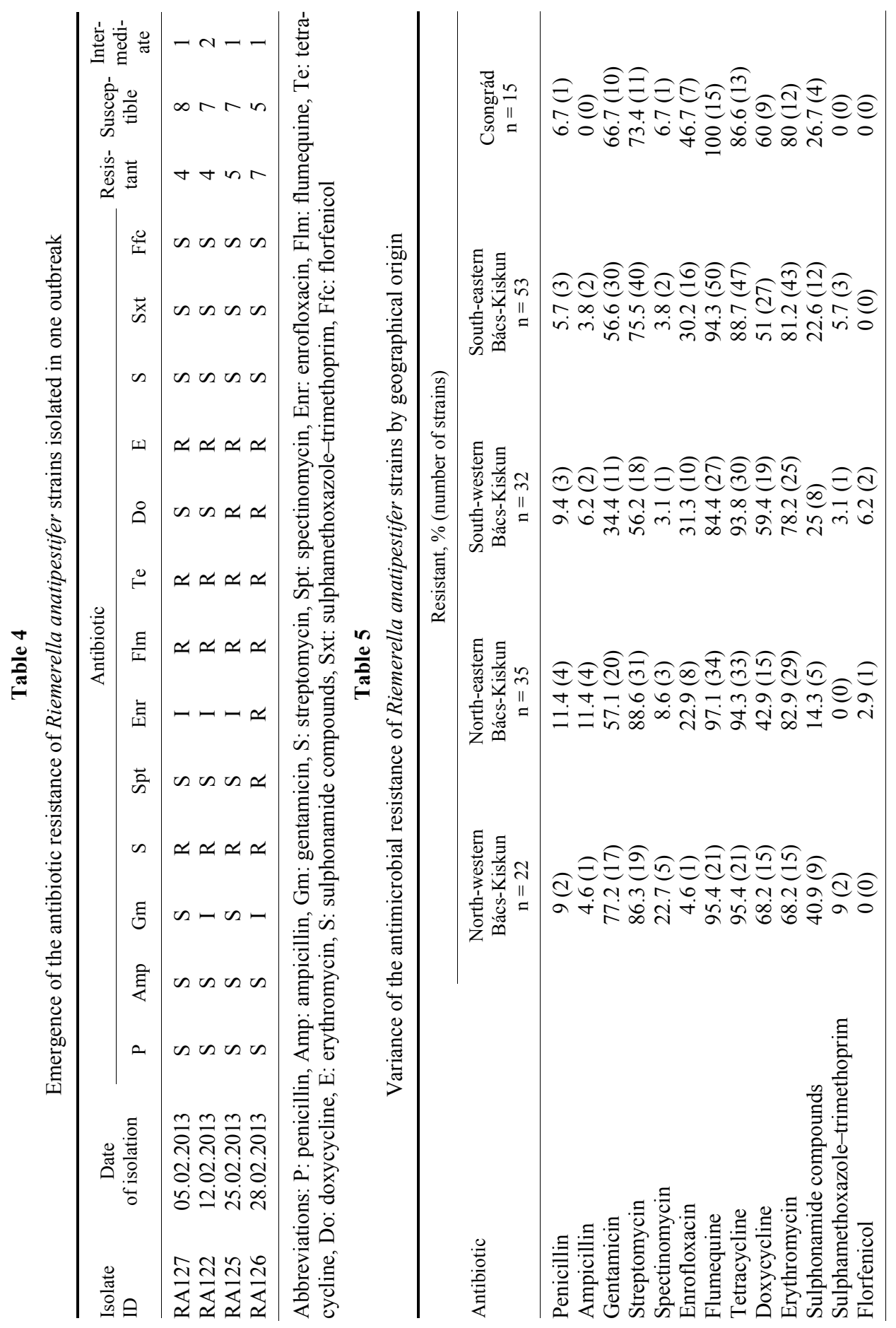




\section{Discussion}

Due to the lack of a vaccine showing cross-protection among different serotypes of $R$. anatipestifer, the frequent change of serotypes in farms and the common presence of more than one serotype in a farm, antibiotics are still important tools for controlling anatipestifer disease. On the other hand, the threat of emergence of antibiotic resistance makes it extremely important to monitor bacterial pathogens for antibiotic susceptibility.

Riemerella anatipestifer strains in this study were generally sensitive to penicillin, with only $7 \%$ of the strains proving to be resistant. Earlier studies reported similar findings in Thailand and Taiwan (Pathanasophon et al., 1994; Chang et al., 2003); however, about five years later $58 \%$ of the strains showed resistance to penicillin in Taiwan (Yu et al., 2008). During the same period, Chinese authors also observed a high $(86.9 \%)$ resistance rate to penicillin (Zhong et al., 2009). Ampicillin was also found effective in this study. Interestingly, isolates in China showed remarkable changes in resistance to this antibiotic over time: $58.4 \%$ of the strains were resistant in 2009 , all strains were sensitive in 2012, and 93\% of the strains were resistant in 2015 (Zhong et al., 2009; Sun et al., 2012; Zheng et al., 2015).

Among aminoglycosides and aminocyclitols, spectinomycin was the most effective agent (only $7 \%$ resistance), while gentamicin and streptomycin showed higher resistance rates $(56.8 \%$ and $71.4 \%$, respectively). Aminoglycosides and aminocyclitols are poorly, or not at all, absorbed from the gut, and thus the oral administration of these antibiotics is not recommended in cases of bacterial septicaemia (Gálfi et al., 2012). Chinese authors observed a similar tendency of resistance to spectinomycin (11.3\%), gentamicin (20.8\%), and streptomycin (42.5\%) (Zhong et al., 2009). At the same time, strains isolated in Taiwan proved to be highly resistant to all these aminoglycosides and aminocyclitols (Chang et al., 2003). German authors also observed over $90 \%$ resistance to gentamicin (Köhler et al., 1995). These findings indicate that there is a variable degree of crossresistance among aminoglycosides, presumably because of the existence of diverse bacterial enzymes that can inactivate these antibiotics.

Twenty-six percent of our $R$. anatipestifer strains were resistant to enrofloxacin (a Class 2 second-generation fluoroquinolone), while a surprisingly high percent of the isolates $(60 \%)$ showed intermediate susceptibility to this agent. Flumequine (a Class 1 second-generation fluoroquinolone) proved to be less effective (94\% resistance). Authors in Taiwan made a similar observation: $83 \%$ of the local strains were susceptible to enrofloxacin, while $81 \%$ of them were resistant to flumequine (Yu et al., 2008). In our study, all but 22 out of the 174 flumequine-resistant strains showed decreased sensitivity to enrofloxacin (there were 47 resistant and 105 intermediately susceptible strains). These data support the observation that a fluoroquinolone of advanced generation such as enrofloxacin 
could break through the resistance to flumequine, although its sensitivity often decreases after a while (Gálfi et al., 2012).

The rate of resistance to tetracycline was high (91.4\%) among the Hungarian strains, while resistance to doxycycline occurred in a lower percentage $(59 \%)$. Strains from Taiwan showed a similar trend: $69 \%$ of the isolates were resistant to tetracycline, whereas only $35 \%$ of the strains proved to be resistant to doxycycline (Yu et al., 2008). Chinese $R$. anatipestifer strains were more sensitive: the ratio of resistance was $46.6 \%$ to tetracycline and $24.4 \%$ to doxycycline (Zhong et al., 2009). This tendency is consistent with the fact that cross-resistance exists among tetracyclines, although some members of this group (like doxycycline or minocycline) can break through this resistance.

Up to $75.1 \%$ of our strains were resistant to erythromycin. The resistance rate in other countries varied: Chinese authors found $32.7 \%$ resistance (Zhong et al., 2009), while authors from Taiwan observed 64\% resistance (Yu et al., 2008).

We found only $1.6 \%$ resistance to florfenicol, in accordance with data reported from China (Sun et al., 2012).

Twenty-seven percent of our strains were resistant to sulphonamide compounds, while only $4.3 \%$ showed resistance to sulphamethoxazole-trimethoprim. Sun et al. (2012) found that Chinese isolates were resistant to sulphonamide, while two thirds of the strains were susceptible to sulphamethoxazole-trimethoprim. These data suggest that the sulphamethoxazole-trimethoprim combination is still effective.

We have also evaluated the changes of antibiotic resistance profiles over time. The rates of resistance to erythromycin, spectinomycin, streptomycin (2000-2014) and sulphonamide compounds (2004-2014) tended to increase. The rate of resistance to other antibiotics showed variable trends. Our results are in accordance with the results of Zhong et al. (2009), who found that the rates of resistance to piperacillin and cefoperazone increased and those to spectinomycin and aztreonam decreased from 1998 to 2005 in China, while no regular patterns were observed for other antibiotics. The authors speculated that the improper use of antibiotics might lead to higher antibiotic resistance rates. Other authors reported similar results, observing a high resistance rate of $R$. anatipestifer isolates from ducks, and explaining it by the overuse and improper application of antibiotics in duck farms (Sun et al., 2012). On the other hand, an increase in susceptibility may occur to antibiotics that had not been used for a while (Zhong et al., 2009).

The average rate of extensive drug resistance was $30.3 \%$. The proportion of extensive drug resistance also tended to increase over time. For strains isolated in 2000, 2004-2006, 2009-2010, 2011, 2012, 2013 and 2014, the rate of extensive drug resistance was $0 \%, 6.6 \%, 38.7 \%, 20.8 \%, 18 \%, 30.5 \%$ and $55.3 \%$, respectively. These results are in harmony with the findings of Köhler et al. (1995), who observed an increasing proportion of multidrug-resistant isolates over time. 
The results obtained for our strains isolated from an outbreak within a three-week period support the assumption that local antibiotic usage may facilitate the emergence of antimicrobial resistance. These strains showed resistance against an increasing number of antibiotics (4 to 7). The history did not provide information about the antibiotics used for therapy; however, resistance emerged to antibiotics commonly used in poultry practice, such as doxycycline, enrofloxacin, gentamicin, and spectinomycin. Although this observation is based on only a few samples, it calls attention to a possible unfavourable side effect of antibiotic usage, the rapid spread of antibiotic resistance. This view is supported by Zhong et al. (2009), who described remarkable differences in resistance patterns that largely depended on antibiotic usage on various farms.

Chang et al. (2003) compared the micro-inhibitory concentrations of kanamycin against strains isolated in Taiwan and in the USA. They found significant differences between the two countries, most likely due to variations in their antibiotic usage. In our study, in conformity with data of the literature, resistance patterns and the proportion of XDR strains showed some correlation with their geographic origin. Again, the most probable reason is the dissimilarity in therapeutic practices.

The World Health Organization has ranked antibiotics according to their relevance in human medicine. The criteria for critically important antibiotics are (1) frequent use in human medicine; (2) an antibiotic that is the only one or one of a few alternative medicines; and (3) evidence of transmission of bacteria or genes to humans from non-human sources. Some agents from these antimicrobial classes belong to critically important antibiotics: aminoglycosides, ansamycins, carbapenems, third and fourth generation cephalosporins, glycopeptides, lipopeptides, macrolides, oxazolidinones, penicillins, quinolones, streptogramins, tetracyclines, and drugs used against mycobacterial diseases. Taking this into consideration when choosing antibiotics for the treatment of food-producing animals may help to maintain the effectiveness of antibiotics relevant in human medicine (Collignon et al., 2009).

The development of antibiotic resistance of pathogenic bacteria and the emergence of multidrug-resistant strains represent a growing threat to animal and human health. The overuse and abuse of antibiotics presumably lead to the selection of resistant bacteria. Our findings as well as data of the literature indicate that the resistance rate of bacterial strains has increased over the last decades. This underlines the significance of reducing the use of antibiotics and the importance of the prudent and correct application of appropriate antibiotics, based on accurate diagnosis and antimicrobial susceptibility testing. 


\section{Acknowledgements}

This project was supported by the Hungarian Scientific Research Fund - OTKA K108632. The authors thank Éva Ivanics, Ákos Thuma, László Makrai, József Nagy, Andrea Tünde Bistyák and Csaba Nemes for supplying some of the strains examined in this study.

\section{References}

Bisgaard, M., Bojesen, A. M. and Christensen, J. P. (2008): Riemerella infections. In: Pattison, M., McMullin, P. F., Bradbury, J. M. and Alexander, D. J. (eds) Poultry Diseases. Elsevier Press, Edinburgh, UK. pp. 172-175.

Bitay, Z., Kovács, Gy., Takács, Gy. and Török, L. (1979): Occurrence of the anatipestifer syndrome of ducks in Hungary (Pasteurella anatipestifer bacteria) [in Hungarian, with English abstract]. Magy. Allatorvosok 34, 747-750.

Breed, R. S., Lessel, E. F. and Heist Clise, E. (1957): Genus I: Pasteurella Trevisan. In: Breed, R. S., Murray, E. G. D. and Smith, N. R. (eds) Bergey's Manual of Determinative Bacteriology. 7th edition. Williams and Wilkins, Baltimore, USA. pp. 395-402.

Bruner, D. W. and Fabricant, J. (1954): A strain of Moraxella anatipestifer (Pfeifferella anatipestifer) isolated from ducks. Cornell Vet. 44, 461-464.

Bruner, D. W., Angstrom, C. I. and Price, J. I. (1970): Pasteurella anatipestifer infection in pheasants. A case report. Cornell Vet. 60, 491-494.

Chang, C. F., Lin, W. H., Yeh, T. M., Chiang, T. S. and Chang, Y. F. (2003): Antimicrobial susceptibility of Riemerella anatipestifer isolated from ducks and the efficacy of ceftiofur treatment. J. Vet. Diagn. Invest. 15, 26-29.

Clinical and Laboratory Standards Institute (2013): Performance Standards for Antimicrobial Susceptibility Testing; Twenty-first Informational Supplement; Informational Supplement. CLSI document M100-S21. Clinical and Laboratory Standards Institute, Wayne, PA.

Clinical and Laboratory Standards Institute (2015): Performance Standards for Antimicrobial Disk and Dilution Susceptibility Tests for Bacteria Isolated From Animals. Third edition. CLSI document VET01S. Clinical and Laboratory Standards Institute, Wayne, PA.

Collignon, P., Powers, J. H., Chiller, T. M., Aidara-Kane, A. and Aarestrup, F. M. (2009): World Health Organization ranking of antimicrobials according to their importance in human medicine: a critical step for developing risk management strategies for the use of antimicrobials in food production animals. Clin. Inf. Dis. 49, 132-141.

Dougherty, E., Saunders, L. Z. and Parsons, E. H. (1955): The pathology of infectious serositis of ducks. Am. J. Pathol. 31, 475-487.

Frommer, A., Bock, R., Inbar, A. and Zemer, S. (1990): Muscovy ducks as a source of Pasteurella anatipestifer infection in turkey flocks. Avian Pathol. 19, 161-163.

Fulton, R. M. and Rimler, R. B. (2010): Epidemiologic investigation of Riemerella anatipestifer in a commercial duck company by serotyping and DNA fingerprinting. Avian Dis. 54, 969-972.

Gálfi, P., Csikó, G. and Jerzsele, Á. (2012): Veterinary Pharmacology III [in Hungarian]. Lecture Notes of the University of Veterinary Science, Robbie-Vet Kft., Budapest, Hungary. pp. 149-237.

Helfer, D. H. and Helmboldt, C. F. (1977): Pasteurella anatipestifer infection in turkeys. Avian Dis. 21, 712-715.

Hendrickson, J. M. and Hilbert, K. F. (1932): A new and serious septicemic disease of young ducks with a description of the causative organism, Pfeifferella anatipestifer. N. S. Cornell Vet. 22, 239-252. 
Hinz, K. H., Ryll, M., Köhler, B. and Glünder, G. (1998): Phenotypic characteristics of Riemerella anatipestifer and similar micro-organisms from various hosts. Avian Pathol. 27, 33-43.

Ivanics, É., Glávits, R. and Édes, I. (1996): A study on the anatipestifer disease of growing geese [in Hungarian, with English abstract]. Magy. Allatorvosok 51, 9-14.

Kardos, G., Nagy, J., Antal, M., Bistyák, A., Tenk, M. and Kiss, I. (2007): Development of a novel PCR assay specific for Riemerella anatipestifer. Lett. Appl. Microbiol. 44, 145-148.

Karstad, L., Lusis, P. and Long, J. R. (1970): Pasteurella anatipestifer as a cause of mortality in captive wild waterfowl. J. Wildl. Dis. 6, 408-413.

Köhler, B., Heiss, R. and Albrecht, K. (1995): Riemerella anatipestifer as pathogen for geese in the northern and central parts of Germany. In: Proceedings of the 49th Symposium on Poultry Diseases of the German Veterinary Medical Society, Hanover. German Veterinary Medical Society Publisher, Giessen, Germany. pp. 57-71.

Magiorakos, A. P., Srinivasan, A., Carey, R. B., Carmeli, Y., Falagas, M. E., Giske, C. G., Harbarth, S., Hindler, J. F., Kahlmeter, G., Olsson-Liljequist, B., Paterson, D. L., Rice, L. B., Stelling, J., Struelens, M. J., Vatopoulos, A., Weber, J. T. and Monnet, D. L. (2012): Multidrug-resistant, extensively drug-resistant and pandrug-resistant bacteria: an international expert proposal for interim standard definitions for acquired resistance. Clin. Microbiol. Infect. 18, 268-281.

Pascucci, S., Giovannetti, L. and Massi, P. (1989): Pasteurella anatipestifer infection in guinea fowl and Japanese quail (Coturnix coturnix japonica). Proceedings of the 9th International Congress of the World Veterinary Poultry Association, Brighton, England. p. 47.

Pathanasophon, P., Tanticharoenyos, T. and Sawada, T. (1994): Physiological characteristics, antimicrobial susceptibility and serotypes of Pasteurella anatipestifer isolated from ducks in Thailand. Vet. Microbiol. 39, 179-185.

Riemer von, S. (1904): Kurze Mitteilung über eine bei Gänsen beobactet exsudative Septikämie und deren Erreger. Zentralbl. Bakteriol. I Abt. I Orig. 37, 641-648.

Rosenfeld, L. E. (1973): Pasteurella anatipestifer infection in fowls in Australia. Aust. Vet. J. 49, $55-56$.

Ruiz, J. A. and Sandhu, T. S. (2013): Riemerella anatipestifer infection. In: Saif, Y. M. (ed.) Diseases of Poultry. Iowa State University Press, Iowa, USA. pp. 823-828.

Segers, P., Mannheim, W., Vancanneyt, M., de Brandt, K., Hinz, K. H., Kersters, K. and Vandamme, P. (1993): Riemerella anatipestifer gen. nov., comb. nov., the causative agent of septicemia anserum exsudativa, and its phylogenetic affiliation within the FlavobacteriumCytophaga rRNA homology group. Int. J. Syst. Bacteriol. 43, 768-776.

Smith, J. M., Frame, D. D., Cooper, G., Bickford, A. A., Ghazikhanian, G. Y. and Kelly, B. J. (1987): Pasteurella anatipestifer infection in commercial meat-type turkeys in California. Avian Dis. 31, 913-917.

Sun, N., Liu, J. H., Yang, F., Lin, D. C., Li, G. H., Chen, Z. L. and Zeng, Z. L. (2012): Molecular characterization of the antimicrobial resistance of Riemerella anatipestifer isolated from ducks. Vet. Microbiol. 158, 376-383.

Yu, C. Y., Liu, Y. W., Chou, S. J., Chao, M. R., Weng, B. C., Tsay, J. G., Chiu, C. H., Wu, C. C., Lin, T. L., Chang, C. C. and Chu, C. (2008): Genomic diversity and molecular differentiation of Riemerella anatipestifer associated with eight outbreaks in five farms. Avian Pathol. 37, 273-279.

Zheng, F., Chen, Q., Gong, X. and Liu, Y. (2015): Comparison of antibiotic resistances of the bacteria harboring and not carrying integrons. J. An. Vet. Adv. 14, 69-74.

Zhong, C. Y., Cheng, A. C., Wang, M. S., Zhu, D. K., Luo, Q. H., Zhong, C. D., Li, L. and Duan, Z. (2009): Antibiotic susceptibility of Riemerella anatipestifer field isolates. Avian Dis. 53, 601-607. 